\title{
Editorial
}

\section{Functional Nanomaterials for Optoelectric Conversion and Energy Storage 2014}

\author{
Yongfeng Luo, ${ }^{1} \mathrm{Li} \mathrm{Li}^{2}$ Sanqing Huang, ${ }^{3}$ Tao Chen, ${ }^{4}$ and Hongmei Luo ${ }^{5}$ \\ ${ }^{1}$ College of Science, Central South University of Forestry and Technology, Changsha, Hunan 410004, China \\ ${ }^{2}$ Shanghai Engineering Research Center of Aquatic-Product Processing \& Preservation, \\ College of Food Science and Technology, Shanghai Ocean University, Shanghai 201306, China \\ ${ }^{3}$ School of Materials and Textiles, Zhejiang Sci-Tech University, Hangzhou, Zhejiang 310018, China \\ ${ }^{4}$ Department of Macromolecular Science and Engineering, Case Western Reserve University, Cleveland, OH 44106, USA \\ ${ }^{5}$ Department of Chemical Engineering, New Mexico State University, Las Cruces, NM 88003, USA \\ Correspondence should be addressed to Yongfeng Luo; yongfengluo@csu.edu.cn
}

Received 23 September 2014; Accepted 23 September 2014; Published 22 December 2014

Copyright (C) 2014 Yongfeng Luo et al. This is an open access article distributed under the Creative Commons Attribution License, which permits unrestricted use, distribution, and reproduction in any medium, provided the original work is properly cited.

With depleting fossil fuels and growing concern on environmental protection, urgent research efforts are needed to find alternative energy resources that are efficient, economically and ecologically friendly. New nanomaterials [1] have opened up new frontiers in materials science and engineering to meet this challenge, particularly functional nanomaterials, for photovoltaic and energy storage. During the past 25 years, therefore, considerable efforts have been made to utilize the unique properties of functional nanomaterials, including carbon nanomaterial $[2,3]$, metallic oxide [4-6], and organic materials [7] as energy materials, and tremendous progress has been achieved in developing high-performance optoelectric conversion and energy storage devices.

Because of the rapid development of optoelectric conversion and energy storage in recent years, we would like to take the opportunity to launch this special issue focusing on advanced functional nanomaterials for optoelectric conversion and energy storage. The aim of this special issue is to highlight remarkable contributions made by the related scientists in this important research area and to cover the broad impacts of energy storage and conversion. This special issue contains 3 review articles and 8 research articles.

Solar cells are the main optoelectric conversion devices, which can efficiently convert sunlight into electricity. This special issue will provide the readers with two review articles and one paper on solar cells. The recent advancement in carbon nanotube fibers in "The carbon nanotube fibers for optoelectric conversion and energy storage" and cellulose nanofibers in "The cellulose nanofibers for optoelectronic conversion and energy storage" for optoelectric conversion and energy storage and the current challenge including low energy conversion efficiency and low stability and future direction of the energy fiber have been finally summarized. Characteristics of dye-sensitized solar cells with graphene/ZnO nanoparticle bilayer structure were studied by C.-H. Hsu et al. in "Enhanced performance of dyesensitized solar cells with graphene/ZnO nanoparticles bilayer structure."

Lithium batteries with high safety, long cycle life, and high rate performance are very important for their applications. The applicable research progress of carbon nanotubes in lithium-ion battery is described in "Progress in application of CNTs in lithium-ion batteries," and its future development is put forward from its two aspects of being not only the anodic conductive reinforcing material and the cathodic energy storage material but also the electrically conductive framework material. Interconnected $\mathrm{LiFePO}_{4}$ /carbon nanoparticles for Li-ion battery cathode have been fabricated by sol-gel method followed by a carbon coating process involving redox reactions in "High cycling performance cathode material: interconnected $\mathrm{LiFePO}_{4}$ /carbon nanoparticles fabricated by sol-gel method." 
Supercapacitors are another group of energy storage devices, which have the features of high power density, long cycling life, and high energy efficiency. Y. Luo et al. in "The carbon nanotube fibers for optoelectric conversion and energy storage" and "The cellulose nanofibers for optoelectronic conversion and energy storage" highlighted the most recent progress in supercapacitors based on carbon nanotube fibers and cellulose nanofibers. Carbon nanotube fibers and cellulose nanofibers have been explored as both electrode materials and electrode additives for developing high-performance supercapacitors which based on CNT fibers recently attracted more attention.

We hope this special issue with a small collection of papers can provide the readers with an overview of the recent progress achieved and the future developing directions in the optoelectric conversion and energy storage field. We sincerely hope that this special issue could provide a valuable reference and perspective for the research community working in this exciting field and inspire many more to enter it.

\section{Acknowledgment}

We would like to thank all the authors who have contributed high-quality peer-reviewed articles that advance state-of-theart research to this special issue.

$$
\begin{array}{r}
\text { Yongfeng Luo } \\
\text { Li Li } \\
\text { Sanqing Huang } \\
\text { Tao Chen } \\
\text { Hongmei Luo }
\end{array}
$$

\section{References}

[1] K. Kalyanasundaram and M. Grätzel, "Themed issue: nanomaterials for energy conversion and storage," Journal of Materials Chemistry, vol. 22, no. 46, pp. 24190-24194, 2012.

[2] L. Dai, D. W. Chang, J.-B. Baek, and W. Lu, "Carbon nanomaterials for advanced energy conversion and storage," Small, vol. 8, no. 8, pp. 1130-1166, 2012.

[3] M. Pumera, "Graphene-based nanomaterials for energy storage," Energy and Environmental Science, vol. 4, no. 3, pp. 668674, 2011.

[4] A. S. Aricò, P. Bruce, B. Scrosati, J. M. Tarascon, and W. van Schalkwijk, "Nanostructured materials for advanced energy conversion and storage devices," Nature Materials, vol. 4, no. 5, pp. 366-377, 2005.

[5] D. Wang, R. Kou, D. Choi et al., "Ternary self-assembly of ordered metal oxide-graphene nanocomposites for electrochemical energy storage," ACS Nano, vol. 4, no. 3, pp. 1587-1595, 2010.

[6] J. Jiang, Y. Li, J. Liu, X. Huang, C. Yuan, and X. W. Lou, "Recent advances in metal oxide-based electrode architecture design for electrochemical energy storage," Advanced Materials, vol. 24, no. 38, pp. 5166-5180, 2012.

[7] H. Dong, H. Zhu, Q. Meng, X. Gong, and W. Hu, "Organic photoresponse materials and devices," Chemical Society Reviews, vol. 41, no. 5, pp. 1754-1808, 2012. 

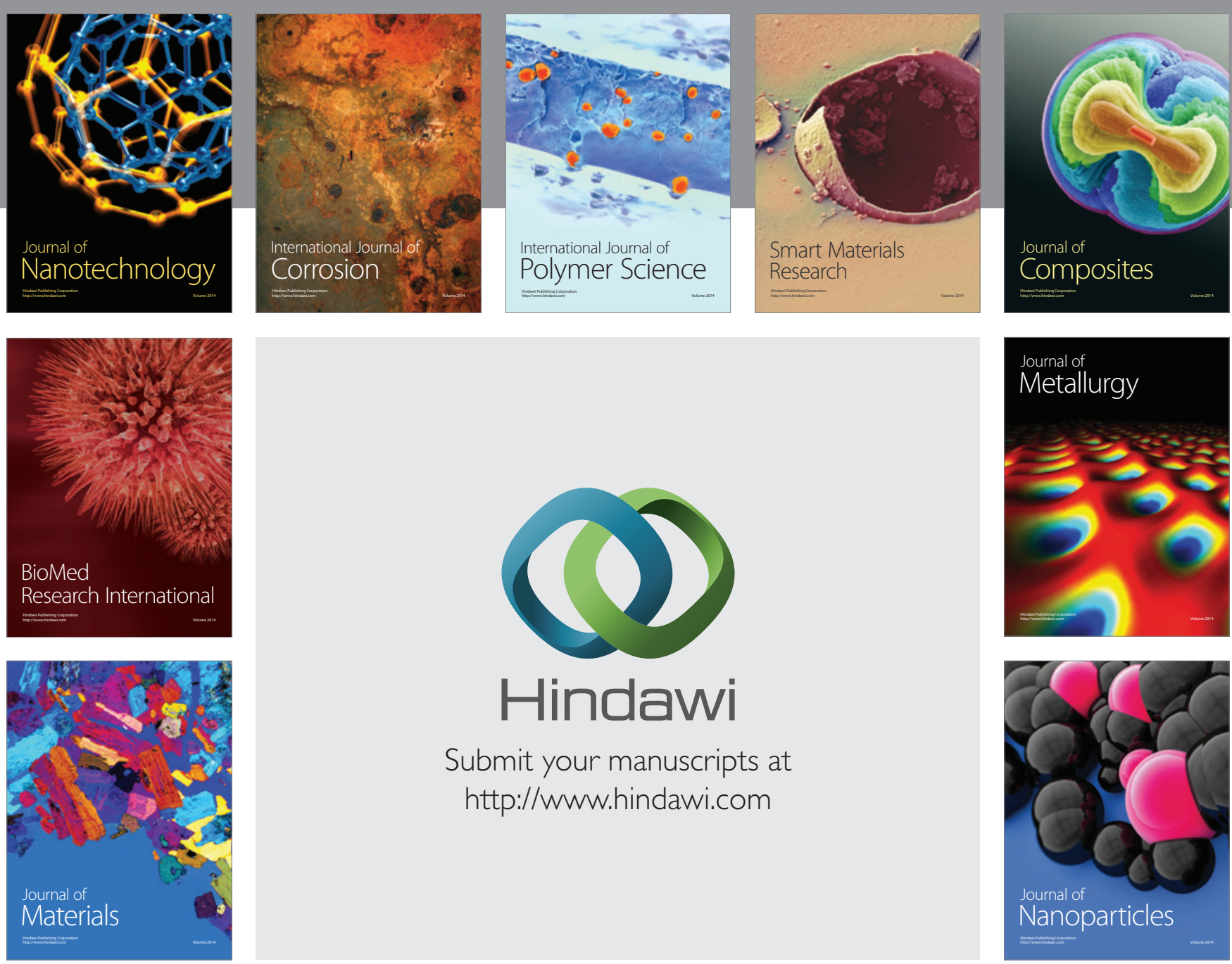

Submit your manuscripts at http://www.hindawi.com
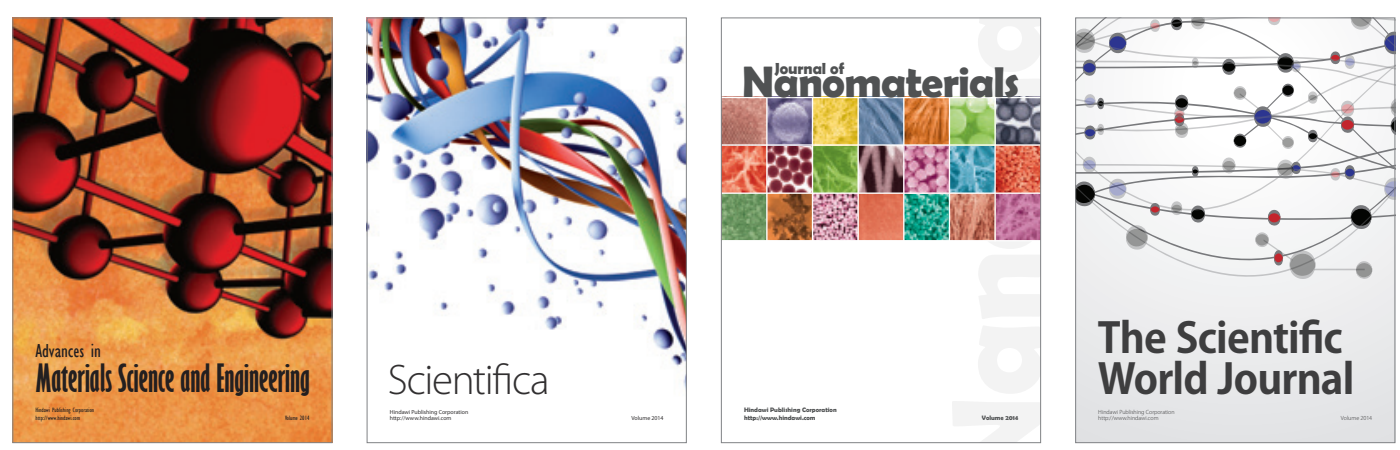

\section{The Scientific World Journal}
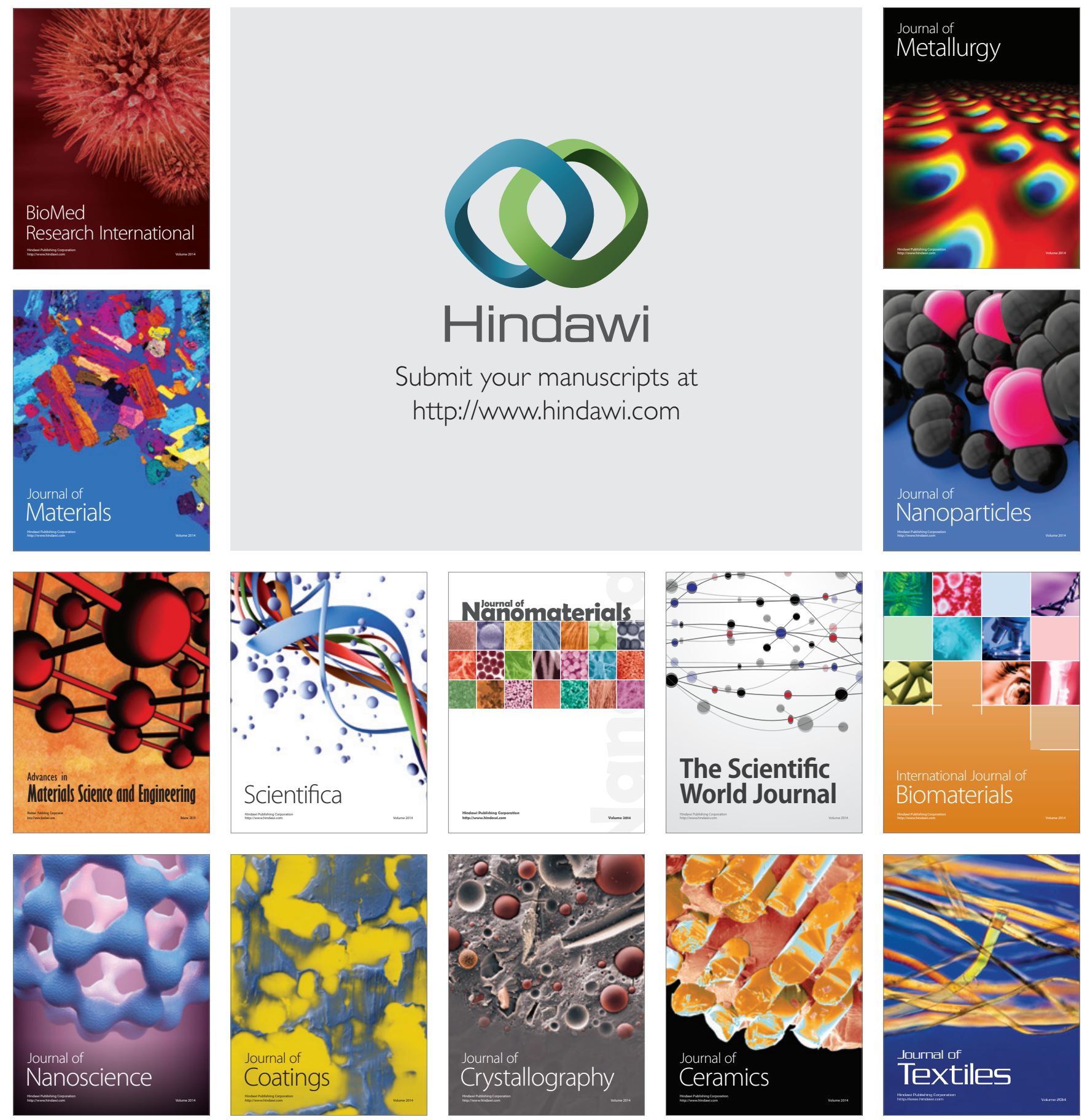